\title{
Cultivo do camarão marinho em água doce em diferentes densidades de estocagem
}

\author{
Sthelio Braga da Fonseca(1), Paulo de Paula Mendes(2), Cândida Juliana de Lyra Albertim ${ }^{(2)}$, \\ Cláudio Figueiroa Bittencourt( ${ }^{(2)}$ e José Humberto Vilar da Silva ${ }^{(1)}$
}

\begin{abstract}
(1)Universidade Federal da Paraíba, Campus III, s/no, Cidade Universitária, CEP 58220-000 Bananeiras, PB. E-mail: sthelio@yahoo.com.br, vilardasiva@yahoo.com.br (2)Universidade Federal Rural de Pernambuco, Departamento de Pesca e Aquicultura, Av. Dom Manuel de Medeiros, s/no, Dois Irmãos, CEP 52171-900 Recife, PE. E-mail: paulo_ufrpe@yahoo.com.br, julialbertim@yahoo.com.br, claudiofbittencourt@yahoo.com.br
\end{abstract}

Resumo - O objetivo deste trabalho foi avaliar o efeito da densidade de estocagem no cultivo do camarão marinho Litopenaeus vannamei, em água doce. Foram avaliadas as seguintes densidades de estocagem: $5\left(\mathrm{D}_{5}\right)$, $10\left(D_{10}\right), 15\left(D_{15}\right), 20\left(D_{20}\right), 25\left(D_{25}\right)$ e $30\left(D_{30}\right)$ camarões por metro quadrado. O delineamento experimental foi inteiramente casualizado, com duas repetições. Foram avaliados o desempenho zootécnico dos camarões e os parâmetros físicos e químicos da água. Os parâmetros zootécnicos de L. vannamei foram estimados por meio de modelos matemáticos. A alcalinidade média da água foi de $36,8 \pm 3,06 \mathrm{mg} \mathrm{L}^{-1}$, e a dureza média de $38,21 \pm 3,07 \mathrm{mg} \mathrm{L}^{-1}$. Aos 52 dias de cultivo, o tratamento $\mathrm{D}_{5}$ foi o que promoveu os melhores resultados em peso $(5,40 \pm 0,99 \mathrm{~g})$ e comprimento $(9,50 \pm 0,20 \mathrm{~cm})$, no entanto, não diferiu significativamente dos tratamentos $D_{10}, D_{15}$ e $D_{25}$ em termos de peso. $O$ tratamento $D_{25}$ teve maior sobrevivência de camarões $(72,10 \pm 9,3 \%)$. Com base nos modelos matemáticos de peso e comprimento em função do tempo de cultivo, concluiu-se que maiores densidades de estocagem proporcionam camarões com menores ganhos de peso e comprimento, em comparação com as menores densidades.

Termos para indexação: Litopenaeus vannamei, aclimatação, alcalinidade, baixa salinidade.

\section{Marine shrimp rearing in fresh water in different stocking densities}

\begin{abstract}
The objective of this work was to evaluate the effect of stocking density in Litopenaeus vannamei rearing in fresh water. The evaluated densities were: $5\left(D_{5}\right), 10\left(D_{10}\right), 15\left(D_{15}\right), 20\left(D_{20}\right), 25\left(D_{25}\right)$ and $30\left(D_{30}\right)$ shrimp per square meter. The experimental design was completely randomized, with two replicates. The performance of shrimp and the physical and chemical parameters of water were evaluated. Mathematical models were generated to estimate the zootechnical parameters of $L$. vannamei. The mean alkalinity of water was $36.8 \pm 3.06 \mathrm{mg} \mathrm{L}^{-1}$, and the mean hardness was $38.21 \pm 3.07 \mathrm{mg} \mathrm{L}^{-1}$. At 52 days of rearing, treatment $\mathrm{D}_{5}$ had the best results on weight $(5.40 \pm 0.99 \mathrm{~g})$ and length $(9.50 \pm 0.20 \mathrm{~cm})$, but the weight did not differ significantly from treatments $\mathrm{D}_{10}, \mathrm{D}_{15}$ and $\mathrm{D}_{25}$. Treatment $\mathrm{D}_{25}$ had the best shrimp survival rate $(72.10 \pm 9.3 \%)$. Based on the mathematical models of weight and length as function of rearing time, it was found that higher stocking densities provide shrimp with less weight and length gain.
\end{abstract}

Index terms: Litopenaeus vannamei, acclimatization, alkalinity, low salinity.

\section{Introdução}

O cultivo de camarões marinhos encontra-se em constante desenvolvimento no mundo, e atualmente consiste na única alternativa para suprir a demanda de camarões gerada pelo aumento no consumo e pela estagnação da produção pesqueira. A carcinicultura tem apresentado crescimento anual superior a $10 \%$ (Waldige \& Caseiro, 2003).

De acordo com a Food and Agriculture Organization of the United Nations (2006), mais de 50 países estão envolvidos nessa atividade, que se estende por todos os continentes, principalmente o asiático, que detém cerca de $88,91 \%$ dos camarões comercializados no mundo. Segundo essa instituição, a produção mundial do camarão marinho cultivado chegou a mais de três milhões de toneladas em 2006 e, entre os cinco principais produtores, encontram-se a China (1.242.385 Mg), Tailândia $(500.800 \mathrm{Mg})$, Vietnã $(349.000 \mathrm{Mg})$, Indonésia (339.803 Mg) e Índia (144.347 Mg). O Brasil ocupou a sétima posição, com a produção de 65 mil toneladas. Entre os peneídeos, destaca-se Litopenaeus vannamei como a espécie mais cultivada 
e de extrema importância na América Latina e Ásia (Rojas \& Alfaro, 2007).

A produção brasileira de camarões marinhos vem crescendo significativamente nos últimos anos, destacando-se a Região Nordeste como principal polo produtor do país (Poersch et al., 2006). Esta região é responsável por aproximadamente $95 \%$ da produção nacional da carcinicultura, por possuir clima quente e favorável ao cultivo durante todos os meses do ano (Rocha et al., 2004).

Atualmente, a maior parte dos cultivos de camarão encontra-se em áreas costeiras, o que aumenta significativamente os custos de implantação de uma fazenda, em razão do elevado valor das terras. Uma alternativa para diminuir custos é o cultivo de espécies marinhas em regiões mais ao interior do país, com água de baixa salinidade em relação à do mar (Sowers et al., 2005).

A espécie L. vannamei é tipicamente eurialina e possui habilidade de tolerar larga variação de salinidade $(0,5-40 \%)$. Tal característica a consagrou como espécie popular para a cultura em baixa salinidade (McGraw et al., 2002; Samocha et al., 2002). Este fato, juntamente com a demanda crescente do mercado internacional por camarão cultivado, resultou no desenvolvimento da carcinicultura em águas de regiões interioranas, o que contribuiu para o desenvolvimento desta atividade em países como Estados Unidos (Arizona, Texas, Alabama e Flórida), Equador, Panamá e Brasil (Figueirêdo et al., 2006).

$\mathrm{O}$ conhecimento das estratégias de manejo empregadas no cultivo é um fator de extrema importância para um bom desenvolvimento de L. vannamei em água com baixa salinidade, pois a densidade de estocagem interfere diretamente no rendimento do cultivo. Segundo Wasielesky Júnior (2000), a densidade de estocagem ideal pode variar de acordo com a estratégia de manejo do cultivo, dos parâmetros ambientais e da espécie cultivada. Björnsson (1994) afirmou que, por afetar diretamente a rentabilidade na aquicultura, a densidade de estocagem é um importante componente para a determinação da viabilidade econômica, já que a maior densidade possibilita menor custo de produção.

O objetivo deste trabalho foi avaliar o efeito da densidade de estocagem no cultivo de camarão marinho L. vannamei em água doce.

\section{Material e Métodos}

O cultivo do camarão marinho $L$. vannamei em água doce foi realizado na Estação de Aquicultura Continental Professor Johei Koike, da Universidade Federal Rural de Pernambuco (UFRPE), entre janeiro e abril de 2008.

Antes do início do cultivo, 20 mil pós-larvas com 8-10 dias, adquiridas de uma larvicultura comercial, foram estocadas em duas caixas de fibra de vidro, com capacidade de $1.000 \mathrm{~L}$ cada uma, providas de aeração, para o processo de aclimatação à água doce.

Após 24 horas de estocagem, iniciou-se o processo de redução da salinidade da água, de $36,4 \%$ para $0,0 \%$. O nível de água das caixas foi aumentado em $50 \%$ do seu volume, o que ocasionou a redução da salinidade na mesma proporção. Esse procedimento foi mantido por 12 dias, tempo necessário para que a salinidade da água das caixas atingisse $0,0 \%$.

Durante o processo de aclimatação das pós-larvas à água doce, utilizou-se alimentação à base de biomassa de artêmia e ração comercial com $45 \%$ de proteína bruta, ofertada diariamente, de forma alternada, em intervalos de duas horas, das 8 às $18 \mathrm{~h}$. Diariamente, as variáveis químicas e físicas da água $(\mathrm{pH}$, oxigênio, salinidade e temperatura) foram monitoradas. Quando o pH atingia níveis inferiores a 7, correções com cal hidratada na proporção de $0,022 \mathrm{~g} \mathrm{~L}^{-1}$ eram realizadas, conforme Mendes et al. (2006).

Antes do início do cultivo, os viveiros foram esvaziados e expostos ao sol pelo período de quatro dias, para a erradicação de organismos indesejáveis e oxidação da matéria orgânica. Para o processo de calagem, foram utilizados $11,5 \mathrm{Mg} \mathrm{ha}^{-1}$ de calcário dolomítico por viveiro, dos quais $50 \%$ foi aplicado na primeira calagem, e o restante aplicado de forma fracionada, semanalmente, no decorrer do cultivo. Juntamente com o calcário, foram adicionados $250 \mathrm{~kg} \mathrm{ha}^{-1}$ de cal hidratada por viveiro, empregada da mesma forma que o calcário. Dois dias após a calagem, foi feita a fertilização dos viveiros com $25 \mathrm{~kg} \mathrm{ha}^{-1} \mathrm{de}$ fertilizante químico à base de N-P-K (20-10-20). O fertilizante foi diluído em água, 48 horas antes de sua aplicação.

Após a aclimatação, a sobrevivência obtida foi de $63,9 \%$. As pós-larvas $(0,02 \pm 0,00 \mathrm{~g}$ e $1,36 \pm 0,33 \mathrm{~cm})$ foram contadas individualmente e estocadas nos viveiros, de acordo com as seguintes densidades: $5\left(D_{5}\right), 10\left(D_{10}\right), 15\left(D_{15}\right), 20\left(D_{20}\right), 25\left(D_{25}\right)$ e $30\left(D_{30}\right)$ 
camarões por metro quadrado. A estocagem das pós-larvas foi realizada em 12 viveiros semi-escavados, com área útil de $60 \mathrm{~m}^{2}(6 \times 10 \mathrm{~m})$ cada um, construídos em terra batida e com sistemas independentes de abastecimento e drenagem. O experimento foi realizado em delineamento inteiramente casualizado, com seis tratamentos e duas repetições cada um.

Durante o cultivo, foi utilizada ração comercial com $35 \%$ de proteína bruta, ofertada a lanço duas vezes ao dia até o $20^{\circ}$ dia e em bandejas de alimentação até o $35^{\circ}$ dia. Entretanto, após o 35 dia de cultivo, em consequência de problemas de competidores pela ração, ela voltou a ser ofertada a lanço até o término do cultivo. Duas vezes por semana foram monitoradas as variáveis físico-químicas da água: $\mathrm{pH}$, oxigênio dissolvido e temperatura. Semanalmente, foram mensuradas as concentrações de alcalinidade total, dureza total, fósforo, potássio e magnésio.

Para avaliar o desempenho zootécnico dos camarões, foram realizadas biometrias quinzenais, com base em 10-15 amostras de camarões, coletadas aleatoriamente. Aos 52 dias de cultivo, foram mensurados: o peso, o comprimento orbital (extremidade do télson ao pedúnculo ocular), e o comprimento da antena de cada camarão amostrado.

Ao término do cultivo, os camarões foram capturados, contados e pesados, para a obtenção do peso final e sobrevivência média em cada viveiro.

Os dados dos parâmetros zootécnicos do camarão foram submetidos à análise de variância, e as médias foram comparadas pelo teste de Tukey, a 5\% de probabilidade.

Para avaliar as relações de peso e comprimento, em função do tempo de cultivo, foram testados vários modelos matemáticos e, para a decisão do melhor a ser utilizado, foram avaliados o coeficiente de determinação $\left(\mathrm{R}^{2}\right)$ e a soma do quadrado do resíduo $\left(\mathrm{SQ}_{\mathrm{res}}\right)$ da análise de variância da regressão. Para relacionar o peso em função do crescimento, foi utilizado o seguinte modelo multiplicativo (Mendes et al., 2006): $\mathrm{W}=\varnothing \mathrm{L}^{\theta}$, em que: W é o peso (g); L é o comprimento (cm); e Ø e $\theta$ são os fatores de condição dos animais.

Para relacionar o comprimento das antenas em função do comprimento orbital, foi utilizado o seguinte modelo: $\mathrm{CA}_{\mathrm{i}}=\beta_{0}+\beta_{1} \mathrm{~L}_{\mathrm{i}}+\varepsilon_{\mathrm{i}}$, em que: $\mathrm{CA}_{\mathrm{i}}$ é o comprimento da antena $(\mathrm{cm}) ; \mathrm{L}_{\mathrm{i}}$ é o comprimento orbital; $\beta_{0}$ e $\beta_{1}$ são parâmetros do modelo; i é a i-ésima observação; e $\varepsilon$ é o erro experimental, com parâmetros da distribuição $\left(0, \sigma^{2}\right)$.

Para a comparação dos modelos de crescimento em peso e comprimento, em função do tempo de cultivo e das diferentes densidades de estocagem, utilizou-se a estatística $\mathrm{W}$ de comparação de modelos, a 5\% de probabilidade, definida em Mendes (1999): $\mathrm{W}=\left\{\left(\mathrm{N}_{1}+\mathrm{N}_{2}\right) \operatorname{Ln}[(\mathrm{SQres} 1,2) /\right.$ $\left.\left.\left(\mathrm{N}_{1}+\mathrm{N}_{2}\right)\right]\right\}-\left\{\mathrm{N}_{1} \operatorname{Ln}\left[\left(\mathrm{SQres}_{1}\right) /\left(\mathrm{N}_{1}\right)\right]\right\}-\left\{\mathrm{N}_{2} \operatorname{Ln}\left[\left(\mathrm{SQres}_{2}\right) /\right.\right.$ $\left.\left.\left(\mathrm{N}_{2}\right)\right]\right\}$, em que: W é a estatística a ser comparada com a distribuição de qui-quadrado; $\mathrm{N}_{1}$ e $\mathrm{N}_{2}$ são os números geradores dos modelos 1 e $2 ; \mathrm{SQ}_{\mathrm{res}}$ é a soma dos quadrados do resíduo da análise de regressão; e Ln é o logaritmo neperiano.

\section{Resultados e Discussão}

Aos 52 dias de cultivo, os camarões do tratamento $\mathrm{D}_{5}$ apresentaram o maior peso médio final $(5,4 \pm 0,99 \mathrm{~g})$, semelhantemente ao obtido por Carneiro et al. (1999), que foi de $5,67 \mathrm{~g}$, ao cultivar L. vannamei em água doce, durante 90 dias. Entretanto, não houve diferença significativa entre o tratamento $D_{5}$ e os tratamentos $D_{10}$, $\mathrm{D}_{15}$ e $\mathrm{D}_{25}$. Os camarões do tratamento $\mathrm{D}_{30}$ apresentaram o menor ganho de peso $(1,82 \pm 0,20 \mathrm{~g})$, o que evidencia a relação de decréscimo do peso em função do aumento da densidade (Tabela 1). Essa mesma relação foi observada por Carvalho (2004) que, ao cultivar L. vannamei em densidades de 20 e 40 camarões por metro quadrado, encontrou o melhor ganho de peso no cultivo de menor densidade.

Tabela 1. Parâmetros zootécnicos do Litopenaeus vannamei, aos 52 dias de cultivo em água doce, em diferentes densidades de estocagem ${ }^{(1)}$.

\begin{tabular}{lccc}
\hline Densidade (camarões $\left.\mathrm{m}^{-2}\right)$ & Comprimento $(\mathrm{cm})$ & Peso $(\mathrm{g})$ & Comprimento antena (cm) \\
\hline 5 & $9,50 \pm 0,45 \mathrm{a}$ & $5,40 \pm 0,99 \mathrm{a}$ & $15,69 \pm 0,88 \mathrm{a}$ \\
10 & $8,24 \pm 1,00 \mathrm{~b}$ & $3,33 \pm 1,29 \mathrm{ab}$ & $13,63 \pm 1,71 \mathrm{a}$ \\
15 & $8,21 \pm 0,11 \mathrm{~b}$ & $3,12 \pm 0,04 \mathrm{ab}$ & $12,94 \pm 0,36 \mathrm{a}$ \\
20 & $6,68 \pm 0,59 \mathrm{c}$ & $2,09 \pm 0,085 \mathrm{~b}$ & $10,75 \pm 0,61 \mathrm{a}$ \\
25 & $7,80 \pm 0,22 \mathrm{~b}$ & $2,29 \pm 0,03 \mathrm{ab}$ & $11,90 \pm 0,55 \mathrm{a}$ \\
30 & $6,84 \pm 0,47 \mathrm{bc}$ & $1,82 \pm 0,2 \mathrm{~b}$ & $17,83 \pm 1,00 \mathrm{a}$ \\
$\mathrm{CV}(\%)$ & 9,97 & 10,20 & $71,08 \pm 1,00 \mathrm{bc}$ \\
\hline
\end{tabular}

(1)Médias \pm desvio-padrão seguidas de letras iguais não diferem entre si pelo teste de Tukey, a 5\% de probabilidade. 
Esses resultados podem ser relacionados ao consumo do alimento natural disponível nos viveiros. Nos cultivos com altas densidades de estocagem, o alimento natural torna-se um fator limitante para o desenvolvimento dos camarões. Nesses casos, os camarões passam a depender exclusivamente do alimento artificial, que nem sempre possui todos os nutrientes necessários para um desenvolvimento satisfatório. Segundo Barbieri Júnior \& Ostrensky Neto (2002), cultivos com densidades superiores a 12 camarões por metro quadrado tendem a esgotar o alimento natural existente nos viveiros. Portanto, quanto maior a densidade de estocagem, menor será a disponibilidade de alimento natural, logo maior será o consumo de alimento artificial. Em tratamentos com menor densidade de estocagem, a quantidade de alimento natural disponível nos viveiros passa a ser suficiente para atender as exigências nutricionais dos camarões e proporciona melhor desenvolvimento e menor dependência do alimento artificial. Nunes et al. (1997) constataram que, em viveiros bem preparados, ricos em alimento natural e com baixas densidades de estocagem, o alimento natural pode ser responsável por até $85 \%$ da dieta desses indivíduos.

Bezerra et al. (2007) constataram que a densidade de estocagem é uma das variáveis que exerce maior influência no peso final de L. vannamei. No entanto, Mendes \& Valença (1999), ao cultivar essa espécie em água doce, nas densidades de 10,15 e 20 camarões por metro quadrado, não encontraram diferença significativa no peso médio final.
Ao avaliar o efeito da densidade de estocagem no comprimento orbital dos camarões, verificou-se que o tratamento $D_{5}$ propiciou o melhor resultado $(9,51 \pm 0,45 \mathrm{~cm})$, enquanto os tratamentos $\mathrm{D}_{20}$ e $\mathrm{D}_{30}$ apresentaram os menores ganhos de comprimento. Almeida et al. (1999), ao cultivar L. vannamei em densidades de 10, 20 e 30 camarões por metro quadrado, obtiveram os maiores ganhos de comprimento na menor densidade de cultivo. Krummenauer et al. (2006) cultivaram, em gaiolas, Farfantepenaeus paulensis com diferentes densidades de estocagem e relataram que o aumento na densidade de estocagem influenciou negativamente o crescimento dessa espécie. Santos \& Mendes (2007) analisaram estatisticamente os dados de 53 cultivos comerciais do L. vannamei e concluíram que a densidade de estocagem é uma das variáveis independentes que mais interfere no resultado final dos cultivos.

Com base nos modelos matemáticos de peso e comprimento em função do tempo de cultivo, gerados para a produção do camarão marinho $L$. vannamei cultivado em água doce (Tabela 2), é possível afirmar que cultivos com maiores densidades de estocagem proporcionam camarões com menores ganhos de peso e comprimento, em comparação aos cultivos que utilizam menores densidades de estocagem. Esses modelos apresentaram valores de $\mathrm{R}^{2}$ (coeficiente de determinação) entre 95,04 e 99,81\%. Para a determinação do peso final e do comprimento final dos camarões em função da densidade de estocagem, foram gerados os seguintes modelos matemáticos: $\mathrm{PF}=-0,1259 \mathrm{DE}+5,2147\left(\mathrm{R}^{2}=80,70 \%\right)$, em que

Tabela 2. Modelos matemáticos do peso (g) e comprimento orbital (cm) em função do tempo (dias), de Litopenaeus vannamei cultivado em água doce, em diferentes densidades de estocagem.

\begin{tabular}{|c|c|c|c|c|c|}
\hline Densidade (camarões $\mathrm{m}^{-2}$ ) & Modelo & $\mathrm{EC}^{(1)}$ & $\mathrm{R}^{2}(\%)$ & $\mathrm{F}$ & $\mathrm{p}(\mathrm{F})$ \\
\hline & \multicolumn{5}{|c|}{ Peso $(\mathrm{P}) \mathrm{x}$ tempo $(\mathrm{T})$} \\
\hline 5 & $\mathrm{P}=0,0183 \mathrm{~T}^{1,41}$ & a & 98,33 & 354,3 & $<0,00001$ \\
\hline 10 & $P=0,0185 T^{1,27}$ & $\mathrm{~b}$ & 97,39 & 223,5 & $<0,00001$ \\
\hline 15 & $\mathrm{P}=0,0192 \mathrm{~T}^{1,26}$ & $\mathrm{c}$ & 99,81 & $3.183,7$ & $<0,00001$ \\
\hline 20 & $\mathrm{P}=0,0191 \mathrm{~T}^{1,18}$ & be & 98,78 & 848,7 & $<0,00001$ \\
\hline 25 & $\mathrm{P}=0,0213 \mathrm{~T}^{1,20}$ & $\mathrm{~b}$ & 98,63 & 432,9 & $<0,00001$ \\
\hline \multirow[t]{2}{*}{30} & $\mathrm{P}=0,0204 \mathrm{~T}^{1,15}$ & de & 99,66 & $1.774,1$ & $<0,00001$ \\
\hline & \multicolumn{5}{|c|}{ Comprimento orbital (C) $\mathrm{x}$ tempo $(\mathrm{T})$} \\
\hline 5 & $\mathrm{C}=1,2854 \mathrm{~T}^{0,47}$ & $\mathrm{a}$ & 97,26 & 213,4 & $<0,00001$ \\
\hline 10 & $\mathrm{C}=1,2785 \mathrm{~T}^{0,43}$ & $a b$ & 95,04 & 114,9 & $<0,00001$ \\
\hline 15 & $\mathrm{C}=1,3012 \mathrm{~T}^{0,43}$ & $a b$ & 97,98 & 290,8 & $<0,00001$ \\
\hline 20 & $\mathrm{C}=1,3225 \mathrm{~T}^{0,39}$ & $\mathrm{~b}$ & 97,80 & 267,3 & $<0,00001$ \\
\hline 25 & $\mathrm{C}=1,3381 \mathrm{~T}^{0,42}$ & $a b$ & 98,16 & 324,1 & $<0,00001$ \\
\hline 30 & $\mathrm{C}=1,3273 \mathrm{~T}^{0,40}$ & $\mathrm{~b}$ & 98,57 & 414,9 & $<0,00001$ \\
\hline
\end{tabular}


PF é o peso final (g) e DE é a densidade de estocagem (camarões $\left.\mathrm{m}^{-2}\right) ; \mathrm{eCF}=-0,0923 \mathrm{DE}+9,498\left(\mathrm{R}^{2}=69,01 \%\right)$, em que CF é o comprimento final $(\mathrm{cm})$.

Em relação à sobrevivência, os camarões do tratamento $\mathrm{D}_{25}$ apresentaram significativamente os melhores resultados $(72,10 \%)$, enquanto os camarões do tratamento $D_{10}$ apresentaram a menor sobrevivência $(18,83 \%)$ (Tabela 1). Os resultados de sobrevivência podem ter sido afetados pelo elevado índice de predadores e competidores encontrados nos viveiros ao término do cultivo. Em razão desse fato, após o $35^{\circ}$ dia de cultivo, a ração deixou de ser ofertada em bandejas e passou a ser ofertada a lanço, como forma de diminuir o consumo de ração pelos competidores. Apesar desse problema, a sobrevivência dos camarões do tratamento $\mathrm{D}_{25}$ foi similar à encontrada por Decamp et al. (2007) que, ao cultivar L. vannamei em água com 36\% de salinidade e em tanques de fibra, em três diferentes densidades, obtiveram a sobrevivência média de $76,7 \%$.

Nas análises da relação peso e comprimento orbital e da relação comprimento orbital e comprimento da antena dos camarões, não foi observada diferença significativa entre as densidades de estocagem (Tabela 3). Vale ressaltar que, na relação peso e comprimento, verificou-se que o fator de condição comprimento $(\theta)$ variou entre 2,81 e 2,98 , portanto próximo de 3 , o que evidencia um crescimento isométrico dos camarões, ou seja, os camarões cresceram em comprimento proporcionalmente ao peso. Ao correlacionar o ganho de comprimento do camarão com o crescimento de sua antena, pode-se observar uma relação direta entre esses parâmetros, ou seja, o aumento no tamanho da antena do camarão é proporcional ao seu ganho de comprimento.

Segundo Valenti (1990), a análise do peso e do comprimento é realizada para estudar a variação do peso esperado para determinado valor de comprimento, como indicador de acúmulo de gordura e de desenvolvimento gonadal. Segundo Mendes et al. (1996), esses parâmetros são de essencial importância biológica, por caracterizar uma relação direta com o grau de engorda e o tipo de crescimento do camarão, mas podem variar em função das condições ambientais e das características genéticas da espécie.

Durante o período experimental, as variáveis físicas e químicas da água mantiveram-se próximo ao ideal para o cultivo de camarões (Tabela 4).

Tabela 3. Modelos matemáticos da relação entre peso $(\mathrm{g})$ e comprimento orbital $(\mathrm{cm})$, e entre comprimento da antena (cm) e comprimento orbital, de Litopenaeus vannamei cultivado em água doce, em diferentes densidades de estocagem.

\begin{tabular}{|c|c|c|c|c|c|}
\hline Densidade (camarões $\mathrm{m}^{-2}$ ) & Modelo & $\mathrm{EC}^{(1)}$ & $\mathrm{R}^{2}(\%)$ & $\mathrm{F}$ & $\mathrm{p}(\mathrm{F})$ \\
\hline & \multicolumn{5}{|c|}{ Peso $(\mathrm{P}) \times$ comprimento orbital $(\mathrm{C})$} \\
\hline 5 & $P=0,0091 C^{2,96}$ & $\mathrm{a}$ & 98,74 & 471,8 & 0,00001 \\
\hline 10 & $P=0,0097 C^{2,87}$ & a & 98,61 & 424,5 & 0,00001 \\
\hline 15 & $P=0,0094 C^{2,87}$ & $\mathrm{a}$ & 98,97 & 577,6 & 0,00001 \\
\hline 20 & $P=0,0086 C^{2,98}$ & $\mathrm{a}$ & 98,75 & 473,9 & 0,00001 \\
\hline 25 & $P=0,0097 C^{2,81}$ & a & 98,35 & 357,5 & 0,00001 \\
\hline \multirow[t]{2}{*}{30} & $\mathrm{P}=0,0094 \mathrm{C}^{2,87}$ & $\mathrm{a}$ & 98,60 & 423,1 & 0,00001 \\
\hline & \multicolumn{5}{|c|}{ Comprimento antena (CA) x comprimento orbital (C) } \\
\hline 5 & $\mathrm{CA}=-1,5749+1,8793 \mathrm{C}$ & $\mathrm{a}$ & 98,73 & 467,4 & 0,00001 \\
\hline 10 & $\mathrm{CA}=-1,6595+1,9018 \mathrm{C}$ & $\mathrm{a}$ & 99,12 & 678,5 & 0,00001 \\
\hline 15 & $\mathrm{CA}=-1,4082+1,8253 \mathrm{C}$ & $\mathrm{a}$ & 97,54 & 238,3 & 0,00001 \\
\hline 20 & $\mathrm{CA}=-1,8687+1,9437 \mathrm{C}$ & a & 98,43 & 376,5 & 0,00001 \\
\hline 25 & $\mathrm{CA}=-1,6360+1,7915 \mathrm{C}$ & a & 98,10 & 309,1 & 0,00001 \\
\hline 30 & $\mathrm{CA}=-1,8246+1,9575 \mathrm{C}$ & $\mathrm{a}$ & 98,44 & 379,1 & 0,00001 \\
\hline
\end{tabular}

${ }^{(1)}$ Modelos seguidos por letras iguais não se diferenciam quanto às densidades de estocagem, pela estatística $\mathrm{W}$, a $5 \%$ de probabilidade. EC, e $\mathrm{C}$, estatística comparativa; $R^{2}$, coeficiente de determinação; F, estatística de Snedecor; $p(F)$, probabilidade de rejeição de $F$.

Tabela 4. Parâmetros da água doce com cultivo de Litopenaeus vannamei, em diferentes densidades de estocagem.

\begin{tabular}{|c|c|c|c|c|c|c|c|c|}
\hline $\begin{array}{l}\text { Densidade } \\
\left(\text { camarões } \mathrm{m}^{-2}\right)\end{array}$ & $\begin{array}{l}\text { Oxigênio dissolvido } \\
\left(\mathrm{mg} \mathrm{L}^{-1}\right)\end{array}$ & $\begin{array}{c}\text { Temperatura } \\
\left({ }^{\circ} \mathrm{C}\right) \\
\end{array}$ & $\mathrm{pH}$ & Alcalinidade & Dureza & $\begin{array}{l}\text { Fósforo } \\
-\left(\mathrm{mg} \mathrm{L}^{-1}\right)-\end{array}$ & Potássio & Magnésio \\
\hline 5 & $3,79 \pm 0,42$ & $29,7 \pm 0,95$ & $6,4 \pm 0,31$ & $47,02 \pm 4,81$ & $47,88 \pm 3,70$ & $0,36 \pm 0,18$ & $2,35 \pm 0,35$ & $5,72 \pm 0,85$ \\
\hline 10 & $4,56 \pm 0,47$ & $28,8 \pm 1,00$ & $6,4 \pm 0,34$ & $41,00 \pm 1,61$ & $41,94 \pm 1,39$ & $0,18 \pm 0,06$ & $2,00 \pm 0,00$ & $4,84 \pm 0,59$ \\
\hline 15 & $4,51 \pm 0,60$ & $29,1 \pm 1,01$ & $6,5 \pm 0,36$ & $32,20 \pm 1,91$ & $34,32 \pm 1,65$ & $0,12 \pm 0,00$ & $2,00 \pm 0,00$ & $4,55 \pm 0,31$ \\
\hline 20 & $3,99 \pm 0,50$ & $29,1 \pm 0,71$ & $6,6 \pm 0,30$ & $34,80 \pm 3,41$ & $36,70 \pm 4,05$ & $0,15 \pm 0,09$ & $2,35 \pm 0,35$ & $5,35 \pm 0,63$ \\
\hline 25 & $3,93 \pm 0,50$ & $28,7 \pm 0,48$ & $6,8 \pm 0,33$ & $40,58+3,03$ & $42,68+4,04$ & $0,18 \pm 0,06$ & $2,00 \pm 0,00$ & $5,00 \pm 0,47$ \\
\hline 30 & $4,28 \pm 0,45$ & $28,9 \pm 0,69$ & $6,7 \pm 0,35$ & $25,2 \pm 3,60$ & $25,72 \pm 3,62$ & $0,09 \pm 0,03$ & $1,80 \pm 0,20$ & $3,02 \pm 0,59$ \\
\hline
\end{tabular}


A temperatura variou dentro da faixa confortável para o L. vannamei, que é de 26 a $33^{\circ} \mathrm{C}$ (Nunes, 2002). $\mathrm{O}$ pH da água de cultivo variou entre 6,14 e 6,8 , valor pouco abaixo do recomendado por Malpartida et al. (2004) que é o $\mathrm{pH}$ próximo à neutralidade.

As concentrações de alcalinidade e dureza totais da água, antes do cultivo, foram inferiores a $10 \mathrm{mg} \mathrm{L}^{-1}$, enquanto as concentrações de fósforo total, potássio e magnésio foram de 0,06, 1,20 e 1,40 $\mathrm{mg} \mathrm{L}^{-1}$, respectivamente (Tabela 4). As maiores concentrações de alcalinidade e dureza foram encontradas no tratamento $\mathrm{D}_{5}\left(47,02 \mathrm{e} 47,88 \mathrm{mg} \mathrm{L}^{-1}\right.$, respectivamente). As menores concentrações foram encontradas no tratamento $\mathrm{D}_{30}$, com valores de alcalinidade e dureza de 25,20 e 25,72, respectivamente. Apesar de Boyde et al. (2002) citarem que para cultivar $L$. vannamei em água doce as concentrações de alcalinidade e dureza da água devem ser superiores a $50 \mathrm{mg} \mathrm{L}^{-1}$, demonstrou-se que é possível cultivar essa espécie em água doce com concentrações inferiores a essa.

\section{Conclusões}

1. As menores densidades de estocagem proporcionam maior ganho de peso e maior comprimento do camarão marinho Litopenaeus vannamei cultivado em água doce.

2. É possível cultivar o camarão marinho, L. vannamei em água doce e com concentrações de alcalinidade e dureza totais entre $25 \mathrm{e}^{2} \mathrm{mg} \mathrm{L}^{-1}$, respectivamente.

\section{Agradecimentos}

À Universidade Federal Rural de Pernambuco, ao Conselho Nacional de Desenvolvimento Científico e Tecnológico e ao Programa Recarcine, da Financiadora de Estudos e Projetos, pela infraestrutura e apoio financeiro.

\section{Referências}

ALMEIDA, S.A.A.; CÉSAR, J.R.O.; IGARASHI, M.A.; BEZERRA, F.J.S.; CARVALHO, M.C. Estudo preliminar do cultivo de Penaeus vannamei (Boone, 1931) em tanques com diferentes densidades de estocagem. In: CONGRESSO BRASILEIRODEENGENHARIADEPESCA, 11.;CONGRESSO LATINO-AMERICANO DE ENGENHARIA DE PESCA, 1., 1999, Recife. Anais. Recife: Associações dos Engenheiros de Pesca de Pernambuco, 1999. v.2, p.648-653.
BARBIERI JÚNIOR, R.C.; OSTRENSKY NETO, A. Camarões marinhos: engorda. Viçosa: Aprenda Fácil, 2002. v.2, 352p.

BEZERRA, A.M.; SILVA, J.A.A. da; MENDES, P. de P. Seleção de variáveis em modelos matemáticos dos parâmetros de cultivo do camarão marinho Litopenaeus vannamei. Pesquisa Agropecuária Brasileira, v.42, p.385-391, 2007.

BJÖRNSSON, B. Effects of stocking density on growth rate of halibut (Hippoglossus hippoglossus) reared in large circular tanks for three years. Aquaculture, v.123, p.259-270, 1994.

BOYD, C.E.; THUNJAI, T.; BOONYARATPALIN, M. Dissolved salts for inland, low-salinity shrimp culture. Global Aquaculture Advocate, v.5, p.40-45, 2002.

CARNEIRO, K.B.; CÉZAR, J.R.O.; ALMEIDA, S.A.A.; BEZERRA, F.J.S.; IGARASHI, M.A. Estudo preliminar de um cultivo em água doce do camarão marinho Litopenaeus vannamei Boone, 1931, em tanques retangulares. In: CONGRESSO BRASILEIRO DE ENGENHARIADE PESCA, 11.; CONGRESSO LATINO-AMERICANO DE ENGENHARIA DE PESCA, 1., 1999, Recife. Anais. Recife: Associações dos Engenheiros de Pesca de Pernambuco, 1999. v.2, p.662-668.

CARVALHO, J.F. As desvantagens das altas densidades de povoamento. Revista Panorama da Aqüicultura, v.14, p.53-58, 2004.

DECAMP, O.; CONQUEST, L.; CODY, J.; FORSTER, I.; ALBERT, G.; TACON, J. Effect of shrimp stocking density on size-fractionated phytoplankton and ecological groups of ciliated protozoa within zero-water exchange shrimp culture systems. Journal of the World Aquaculture Society, v.38, p.395-406, 2007.

FIGUEIRÊDO, M.C.B. de; ARAÚJO, L.F.P. de; ROSA, M.F. de; MORAIS, L.F.S. de; PAULINO, W.D.; GOMES, R.B. Impactos ambientais da carcinicultura de águas interiores. Engenharia Sanitária e Ambiental, v.11, p.231-240, 2006.

FOOD AND AGRICULTURE ORGANIZATION OF THE UNITED NATIONS. FishStat plus: universal software for fishery statistical time series. Version 2.3. Rome: FAO, 2006.

KRUMMENAUER, D.; WASIELESKY JÚNIOR, W.; CAVALLI, R.O.; PEIXOTO, S.; ZOGBI, P.R. Viabilidade do cultivo do camarão-rosa Farfantepenaeus paulensis (Crustácea, Decapoda) em gaiolas sob diferentes densidades durante o outono no Sul do Brasil. Ciência Rural, v.36, p.252-257, 2006.

MALPARTIDA, J.; VINATEA, L.; SEIFFERT, W.; BELTRAME, E. Qualidade do solo pode prevenir enfermidades. Revista Panorama da Aqüicultura, v.86, p.53-56, 2004.

MCGRAW, W.J.; DAVIS, D.A.; TEICHERT-CODDINGTON, D.; ROUSE, D.B. Acclimation of Litopenaeus vannamei postlarvae to low salinity: influence of age, salinity endpoint, and rate of salinity reduction. Journal of the World Aquaculture Society, v.33, p.78-84, 2002.

MENDES, G.N.; VALENÇA, A.R. Cultivo de Litopenaeus vannamei em água doce. In: CONGRESSO BRASILEIRO DE ENGENHARIA DE PESCA, 11.; CONGRESSO LATINO-AMERICANO DE ENGENHARIA DE PESCA, 1., 1999, Recife. Anais. Recife: 
Associações dos Engenheiros de Pesca de Pernambuco, 1999. v.2, p.745-749.

MENDES, P. de P.; MENDES, E.S.; BEZERRA, A.M. Análise estatística dos parâmetros aqüícolas, com fins a otimização da produção. Revista Brasileira de Zootecnia, v.35, p.886-903, 2006.

MENDES, P. de P.; SANTOS, J.P.; QUEIROZ, M.F.F. Cultivo do Macrobrachium rosenbergii (de Man, 1879), em tanques com $45 \mathrm{~cm}$ de profundidade. Trabalhos Oceanográficos, v.24, p.229-236, 1996.

MENDES, P. de P. Estatística aplicada à aqüicultura. Recife: Bagaço, 1999. 265p.

MENDES, P. de P.; ALBUQUERQUE, M.L.L.T.; QUEIROZ, D.M. de; SANTOS, B.L. da S.; LIMA, A. da C.; LOPES, Y.V. de A. Aclimatação do camarão marinho Litopenaeus vannamei (Boone, 1931) à água doce com diferentes estratégias de alimentação e calagem. Acta Scientiarum, v.28, p.89-95, 2006.

NUNES, A.J.P. Tratamento de efluentes e recirculação de água na engorda de camarão marinho. Revista Panorama da Aqüicultura, v.12, p.27-39, 2002.

NUNES, A.J.P.; GESTEIRA, T.C.V; GODDARD, S. Food ingestion and assimilation by the southern brown shrimp Penaeus subtilis under semi-intensive culture in NE Brazil. Aquaculture, v.149, p.121-136, 1997.

POERSCH, L.; CAVALLI, R.O.; WASIELESKY JÚNIOR, W.; CASTELlO, J.P.; PEIXOTO S.R.M. Perspectivas para o desenvolvimento dos cultivos de camarões marinhos no estuário da Lagoa dos Patos, RS. Ciência Rural, v.36, p.1337-1343, 2006.

ROCHA, I. de P.; RODRIGUES, J.; AMORIN, L. A carcinicultura brasileira em 2003. Revista da Associação Brasileira de Criadores de Camarão, v.6, p.30-36, 2004.
ROJAS, E.; ALFARO, J. In vitro manipulation of egg activation in the open thelycum shrimp Litopenaeus. Aquaculture, v.264, p.469-474, 2007.

SAMOCHA, T.M.; HAMPER, L.; EMBERSON, C.R.; DAVIS, A.D.; MCINTOSH, D.; LAWRENCE, A.L.; VAN WYK, P.M. Review of some recent developments in sustainable shrimp farming practices in Texas, Arizona, and Florida. Journal of Applied Aquaculture, v.12, p.1-30, 2002.

SANTOS, B.L.S.; MENDES, P.P. de. Análise estatística das variáveis de cultivo do camarão-cinza Litopenaeus vannamei (Boone, 1931). Revista Brasileira de Engenharia de Pesca, v.2, p.128-142, 2007.

SOWERS, A.D.; GATLIN, D.M.; YOUNG, S.P.; ISLEY, J.J.; BROWDY, C.L.; TOMASSO, J.R. Responses of Litopenaeus vannamei (Boone) in water containing low concentrations of total dissolved solids. Aquaculture Research, v.36, p.819-823, 2005.

VALENTI, W.C. Criação de camarões de água doce (Macrobrachium rosenbergii). In: REUNIÃO DA ASSOCIAÇÃO LATINOAMERICANA DE PRODUÇÃO ANIMAL, 22.; REUNIÃO ANUAL DA SOCIEDADE BRASILEIRA DE ZOOTECNIA, 27., 1990, Campinas. Anais. Campinas: Sociedade Brasileira de Zootecnia, 1990. p.757-785.

WALDIGE, V.; CASEIRO, A. Indústria de ração: a situação atual e as perspectivas. Revista Panorama da Aqüicultura, v.13, p.37-45, 2003.

WASIELESKY JÚNIOR, W. Cultivo de juvenis do camarão-rosa Farfantepenaeus paulensis (Decapoda, Penaeidae) no estuário da Lagoa dos Patos: efeitos dos parâmetros ambientais. 2000. 199p. Tese (Doutorado) - Fundação Universidade Federal do Rio Grande, Rio Grande.

Recebido em 29 de dezembro de 2008 e aprovado em 22 de agosto de 2009 